\title{
Frecuencia Cardiaca y su Variabilidad en Caninos de Agility en Gran Altura
}

\author{
Heart rate and its Variability in Agility Dogs at High Altitude \\ Pedro Vargas-Pinto ${ }^{1,3}$, Susana Arango ${ }^{1,4}$, Vanesa Rodríguez ${ }^{1}$, Javier Rivas ${ }^{1}$, \\ Piero Vargas-Pinto ${ }^{2}$
}

\section{Resumen}

El objetivo del presente estudio fue determinar el efecto de la práctica de Agility en caninos en zonas de gran altura $(2650 \mathrm{msnm})$ sobre la frecuencia cardiaca y su variabilidad. Diez caninos Border Collie practicantes de Agility (grupo Agility) y diez caninos sedentarios de iguales características (grupo sedentario) fueron evaluados mediante electrocardiograma en reposo, calentamiento, ejercicio sub-máximo (velocidad máxima de $5 \mathrm{~km} / \mathrm{h}$ por $3 \mathrm{~min}$ ) y descanso en trotadora eléctrica. Se comparó la frecuencia cardiaca en reposo, calentamiento, máxima alcanzada, en descanso y la variabilidad de la frecuencia cardiaca mediante dominio de frecuencia en reposo y descanso. El grupo Agility presentó frecuencias cardiacas menores en todos los puntos de medición $(\mathrm{p}<0.05)$, excepto al comienzo del descanso. El grupo Agility presentó mayores componentes de alta frecuencia en reposo y ejercicio $(\mathrm{p}<0.05)$. Los caninos practicantes de Agility parecen tener una mayor actividad parasimpática en reposo y después de ejercicio sub-máximo.

Palabras clave: frecuencia cardiaca, variabilidad de la frecuencia cardiaca, Agility, gran altura

\section{AbSTRaCT}

The aim of this study was to determine the effect of the practice of dog Agility in high altitude (2550 $\mathrm{m}$ above the sea level) on heart rate and its variability. Ten Agility Border Collie dogs (Agility group) and ten sedentary Border Collie dogs (Sedentary group) were evaluated by electrocardiogram at baseline, warm-up, sub-maximal exercise

${ }^{1}$ Facultad de Ciencias Agropecuarias, Universidad de La Salle, Bogotá, Colombia

${ }^{2}$ Facultad de Medicina Veterinaria y de Zootecnia, Universidad Nacional de Colombia

${ }^{3}$ E-mail: pavargas@unisalle.edu.co

${ }^{4}$ E-mail: pivarvet@hotmail.com

Recibido: 8 de julio de 2016

Aceptado para publicación: 20 de enero de 2017 
(maximum speed of $5 \mathrm{~km} / \mathrm{h}$ for $3 \mathrm{~min}$ ) and rest in an electric treadmill. Heart rate was compared for all time points among groups. Heart rate variability was compared by frequency domain for baseline and at rest. The Agility group had lower heart rates at all time points $(\mathrm{p}<0.05)$ except at the beginning of the rest period. The agility group showed higher high frequency components at baseline and rest $(\mathrm{p}<0.05)$.

Key words: heart rate, heart rate variability, Agility, high altitude

\section{INTRODUCCIÓN}

El entrenamiento físico y la práctica deportiva se consideran como posibles responsables de cambios fisiológicos y patológicos en la estructura y función cardiovascular en humanos (Constable et al., 1994; Karjalainen et al., 1997), de allí que es importante tener en consideración sus posibles consecuencias cuando estas actividades se realizan en condiciones de gran altitud (Stembridge et al., 2015, 2016). Estos fenómenos adaptativos pueden llegar a incrementar el rendimiento físico del individuo, pero son también causas potenciales de disturbios eléctricos (Billman y Kukielka, 2006) o mecánicos (Fagard et al., 1984; Karjalainen et al., 1997), siendo esta última la alteración más común en atletas jóvenes. Sin embargo, cada deporte y su manera de ser practicado producen diferentes efectos en el cuerpo de quien lo practica.

La práctica de deportes por caninos en los Andes suramericanos ha ganado gran popularidad, especialmente el «Agility» (nombre dado en inglés). Este es un deporte internacional de habilidad y destreza donde los perros, conducidos por sus guías, son capaces de superar diversos obstáculos (15 a 20) a gran velocidad, similar a las competencias ecuestres.

Se han realizado diversos estudios en caninos, aunque comparativamente en menor número que en otras especies, siendo mayormente referidos a la práctica de dos deportes concretos: las competiciones de tri- neo y las carreras de galgos (Stepien et al., 1998). Los estudios hechos en Agility en zonas de altura han sido enfocados a los efectos de práctica sobre el estado ácido-base y valores hematológicos (Forero López et al., 2006; Posada Arias et al., 2013). El presente estudio buscó establecer los efectos de la práctica de Agility en gran altura (2650 metros sobre el nivel del mar) sobre el control autonómico del corazón, determinado por cambios en frecuencia cardiaca y variabilidad de la frecuencia cardiaca (Billman y Kukielka, 2006).

\section{Materiales y Métodos}

El protocolo de trabajo del estudio fue aprobado por el Comité de Bioética de la Universidad de La Salle (Acta N. ${ }^{\circ}$ 124). El estudio se llevó a cabo en la ciudad de Bogotá, Colombia, a $2650 \mathrm{msnm}$.

Se trabajó con un grupo de perros de la categoría más alta de Agility en Colombia y que se encontraran entrenando en las fechas del muestreo. Se hizo una evaluación inicial a 15 perros Border Collie, con edades entre 3 y 8 años, de ambos sexos, no gestantes y considerados clínicamente sanos. Cinco de estos animales no lograron adaptarse a la prueba de caminar sobre una trotadora eléctrica a diferentes velocidades, de allí que el grupo quedó conformado por 10 perros (grupo Agility). El grupo control fue conformado por 10 perros de la misma raza, edad y condiciones de salud, pero que no practicaban algún tipo de actividad física regular (grupo sedentarios). 


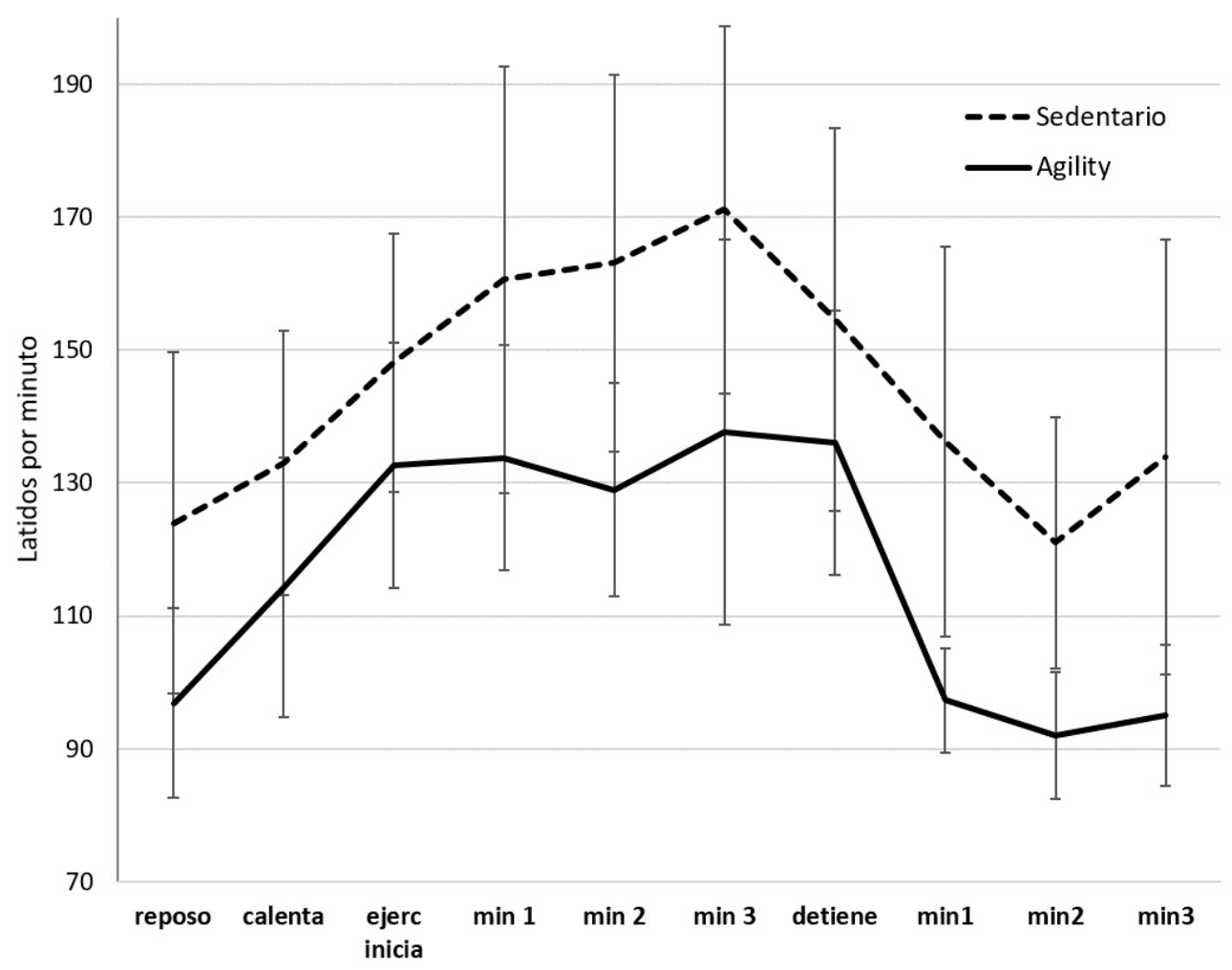

Figura 1. Diferencias en frecuencia cardiaca entre caninos practicantes de Agiliy (ejercicio) y sedentarios (no ejercicio) (Bogotá, Colombia, $2650 \mathrm{msnm}$ ). Se observa, además, las diferencias en tiempo para alcanzar frecuencias máximas y regresar a frecuencia basal. Calenta $=$ calentamiento; ejerc inicia $=$ inicio de ejercicio; detiene $=$ fin del ejercicio

Los animales fueron conectados a un sistema de captación de datos Biopac MP36 (EEUU), conectando los electrodos en el tórax para reducir los efectos de ruido por el movimiento. Los perros fueron colocados de pie sobre una trotadora eléctrica apagada, a fin de capturar la línea base (reposo) de electrocardiograma (ECG) en derivada I, entre 3 y 5 minutos, dependiendo de la colaboración del animal. Se pasó al trabajo de calentamiento en trotadora y rápidamente se pasó a velocidad máxima (de 0 a $5 \mathrm{~km} / \mathrm{h}$ en un minuto), sin inclinación. Tres minutos después se detuvo la trotadora y se siguió capturando datos por tres minutos más (descanso). Se estableció $5 \mathrm{~km} / \mathrm{h}$ como velocidad máxima debido a que la mayoría de los animales no lograba mantenerse en pie a velocidades mayores.
Los ECG fueron analizados mediante el software AcqKnowledge ${ }^{\circledR}$ para duración de intervalo R-R y frecuencia cardiaca instantánea. Se tomaron los puntos de medición en 10 latidos (frecuencia cardiaca) al final de línea base (reposo), inicio de calentamiento, inicio de ejercicio, minuto 1 , minuto 2 y minuto 3 , al detener la trotadora y minutos 1,2 y 3 de descanso. Se obtuvo el promedio y desviación estándar de cada punto de medición para los dos grupos evaluados (Agility y sedentarios). Se realizó la prueba de t-Student para comparaciones en cada uno de los puntos de medición entre los dos grupos.

Se obtuvo el análisis de poder espectral del electrocardiograma completo en los puntos de reposo y descanso utilizando 
Cuadro 1. Resumen del análisis de poder espectral del electrocardiograma en perros entrenados para Agility $(n=10)$ y perros sedentarios $(n=10)$ en una prueba de ejercicio sobre una trotadora eléctrica (Bogotá, Colombia, $2650 \mathrm{msnm}$ )

\begin{tabular}{lccc}
\hline & Agility & Sedentario & Valor de p \\
\hline En reposo - HF $\left(\operatorname{ln~ms}^{2}\right)$ & $1.09 \pm 1.16$ & $0.26 \pm 0.34$ & $<0.05$ \\
En descanso - HF $\left(\operatorname{ln~ms}^{2}\right)$ & $0.92 \pm 0.17$ & $0.21 \pm 0.27$ & $<0.05$ \\
En descanso - LF $(\operatorname{ln~ms})^{2}$ & $0.33 \pm 0.33$ & $0.03 \pm 0.04$ & $<0.05$ \\
\hline
\end{tabular}

Diferencias en las mediciones de dominio de frecuencia. HF muestra los componentes de alta frecuencia en reposo y descanso. LF muestra los componentes de baja frecuencia en descanso

AcqKnowledge ${ }^{\circledR}$. No se realizó este proceso durante el ejercicio debido al movimiento de la línea base. Este análisis permitió la medición de componentes de alta (HF 0.15-0.4 $\mathrm{Hz}$ ) y baja (LF $0.04-0.15 \mathrm{~Hz}$ ) frecuencia del ECG. Los datos fueron promediados y se utilizó la prueba de t-Student para comparación entre grupos utilizando Excel.

\section{Resultados}

La frecuencia cardiaca (FC) fue significativamente diferente en reposo, en ejercicio y en descanso en la comparación entre grupos. La Figura 1 muestra que la FC en el grupo Agility es más baja en estos puntos en comparación con el grupo de sedentarios $(\mathrm{p}<0.05)$, excepto cuando se detiene la trotadora. Si bien esto era esperable, también se observa diferencia en el tiempo promedio en que se alcanza la FC máxima para cada grupo.

Los caninos deportistas practicantes de Agility alcanzaron frecuencias cardiacas máximas más bajas que caninos similares, pero considerados sedentarios. Asimismo, esta baja frecuencia cardiaca máxima (entre 132 a 137 latidos por minuto) fue alcanzada en menor tiempo. El grupo de sedentarios alcanzó una frecuencia cardiaca máxima más alta y en mayor tiempo (entre 160 a 171 latidos por minuto).
El grupo Agility recuperó su frecuencia cardiaca basal en un minuto en promedio. Los perros sedentarios lo lograron en el doble del tiempo después de haber detenido la trotadora (Figura 1).

Los componentes de HF fueron significativamente más altos en reposo y en descanso en el grupo Agility en comparación con el grupo sedentarios $(\mathrm{p}<0.05)$. Asimismo, los componentes de baja frecuencia fueron también más altos en el grupo Agility en comparación con los sedentarios durante el descanso de ejercicio (Cuadro 1).

\section{Discusión}

Las diferencias en frecuencia cardiaca entre los grupos evaluados podrían estar relacionadas a diferencias en la preparación del sistema simpático al inicio del ejercicio, lo que determina que los animales deportistas se encuentren «más preparados» para responder a los nuevos requerimientos metabólicos; asimismo, está asociado a un menor riesgo de muerte por eventos cardiovasculares (Billman y Kukielka, 2006). Esto, no obstante, ha sido indicado como una posible predisposición al desarrollo de arritmias en animales deportistas (Schwartz y Stone, 1982). 
Los caninos del grupo Agility regresaron a su frecuencia cardiaca basal más rápido que el grupo sedentario. Esto sugiere un tono parasimpático más alto y mejor adaptación cardiovascular para el ejercicio con menor riesgo cardiovascular por mejor estabilidad eléctrica cardiaca dada por un control autonómico correcto (Billman y Kukielka, 2006). Otros estudios han demostrado disminución de la variabilidad de la frecuencia cardiaca con aumento de componentes de baja frecuencia, justo antes de iniciar taquicardias ventriculares en pacientes ((Shusterman et $a l ., 1998)$. En este estudio, basado en el análisis espectral, se observó una mayor cantidad de componentes de alta frecuencia en los animales deportistas en los dos puntos de medición. Esto puede deberse al mayor tono parasimpático presente en individuos deportistas (Carter et al., 2003), pero al mismo tiempo, demuestra que en animales deportistas se contrarresta los efectos que genera la hipoxia sobre el sistema simpático y sobre la actividad de barorreceptores (Fletcher, 2000; Hainsworth et al., 2007), generando una actividad autonómica que podría ser cardioprotectora.

Estos resultados se ven complementados con el hallazgo de que los componentes de baja frecuencia fueron mayores en descanso (es decir, justo después de ejercicio submáximo) en los no deportistas, sugiriendo un aumento de la actividad simpática o mayor dificultad para controlar la actividad simpática y que la parasimpática se le sobreponga. Sin embargo, los deportistas también presentaron mayor actividad de baja frecuencia en descanso. Esto sugiere mayor actividad simpática y se relaciona mucho con la pendiente de ascenso de la frecuencia cardiaca y se ha sugerido como una muestra de «mayor preparación» del sistema simpático para ejercicio (Mohan et al., 2000). Estos resultados son preliminares en el establecimiento y variabilidad de la frecuencia cardiaca en animales deportistas en gran altura comparados con animales sedentarios. Dejan a su vez abiertas varias puertas de investigación en variabilidad de la frecuencia cardiaca en animales deportistas en gran altura.

\section{Agradecimiento}

Los autores agradecen a la Vicerrectoría de Investigación y Transferencia de la Universidad de La Salle, por las facilidades prestadas para el desarrollo de la presente investigación.

\section{Literatura Citada}

1. Billman GE, Kukielka M. 2006. Effects of endurance exercise training on heart rate variability and susceptibility to sudden cardiac death: protection is not due to enhanced cardiac vagal regulation. JAppl Physiol 100: 896-906. doi: 10.1152/ japplphysiol.01328.2005

2. Carter JB, Banister EW, Blaber AP. 2003. Effect of endurance exercise on autonomic control of heart rate. Sports Med 33:33-46. doi: 10.2165/00007256200333010-00003

3. Constable PD, Hinchcliff $K W$, Olson J, Hamlin RL. 1994. Athletic heart syndrome in dogs competing in a longdistance sled race. J Appl Physiol 76: 433438.

4. Fagard R, Aubert A, Staessen J, Eynde EV, Vanhees L, Amery A. 1984. Cardiac structure and function in cyclists and runners. Comparative echocardiographic study. Br Heart J 52: 124-129.

5. Fletcher EC. 2000. Effect of episodic hypoxia on sympathetic activity and blood pressure. Respir Physiol 119: 189197. doi: 10.1016/S0034-5687(99)00114-0

6. Forero López JH, Lozano Martínez PA, Camargo Roncancio BO. 2006. Parámetros fisiológicos en caninos pre y post competencia de Agility en Bogotá, Colombia. Rev Med Vet 12: 57-71. doi: 10.19052/mv.2053 
7. Hainsworth R, Drinkhill MJ, RiveraChira M. 2007. The autonomic nervous system at high altitude. Clin Auton Res 17: 13-19. doi: 10.1007/s10286-006-0395-7

8. Karjalainen $J$, Mantysaari M, Viitasalo M, Kujala U. 1997. Left ventricular mass, geometry, and filling in endurance athletes: association with exercise blood pressure. J Appl Physiol 82:531-537.

9. Mohan RM, Choate JK, Golding S, Herring N, Casadei B, Paterson DJ. 2000. Peripheral pre-synaptic pathway reduces the heart rate response to sympathetic activation following exercise training: role of NO. Cardiovasc Res 47: 90-98. doi: 10.1016/S00086363(00)00066-3

10. Posada Arias S, García Naranjo R, Saldarriaga Restrepo A. 2013. Valores hematológicos pre y postejercicio por sexo y por edad en caninos que practican agility en Antioquia. Rev Med Vet 25: 49-62. doi: http://dx.doi.org/10.19052/ mv.2298

11. Schwartz PJ, Stone HL. 1982. The role of the autonomic nervous system in sudden coronary death. Ann N Y Acad
Sci 382: 162-180. doi: 10.1111/j.17496632.1982.tb55214.x

12. Shusterman V, Aysin B, Gottipaty V, Weiss $R$, Brode S, Schwartzman D, Anderson KP. 1998. Autonomic nervous system activity and the spontaneous initiation of ventricular tachycardia. J Am Coll Cardiol 32: 1891-1899. doi: 10.1016/ S0735-1097(98)00468-9

13. Stembridge M, Ainslie PN, Hughes MG, Stohr EJ, Cotter JD, Tymko MM, Day TA, et al. 2015. Impaired myocardial function does not explain reduced left ventricular filling and stroke volume at rest or during exercise at high altitude. J Appl Physiol 119: 1219-1227. doi: 10.1152/japplphysiol.00995.2014

14. Stembridge M, Ainslie PN, Shave R. 2016. Mechanisms underlying reductions in stroke volume at rest and during exercise at high altitude. Eur J Sport Sci 16: 577-584. doi: 10.1080/ 17461391.2015.1071876

15. Stepien RL, Hinchcliff KW, Constable PD, Olson J. 1998. Effect of endurance training on cardiac morphology in Alaskan sled dogs. J Appl Physiol 85: 1368-1375. 